\title{
Maximal service area problem for optimal siting of emergency facilities
}

\begin{abstract}
Geographic information systems (GIS) have been integrated to many applications in facility location problems today. However, there are still some GIS capabilities yet to be explored thoroughly. This study utilizes the capability of GIS to generate service areas as the travel time zones in a facility location model called the maximal service area problem (MSAP). The model is addressed to emergency facilities for which accessibility is an important requirement. The objective of the MSAP is to maximize the total service area of a specified number of facilities. In the MSAP, continuous space is deemed as the demand area, thus the optimality was measured by how large the area could be served by a set of facilities. Fire stations in South Jakarta, Indonesia, were chosen as a case study. Three heuristics, genetic algorithm (GA), tabu search (TS) and simulated annealing (SA), were applied to solve the optimization problem of the MSAP. The final output of the study shows that the three heuristics managed to provide better coverage than the existing coverage with the same number of fire stations within the same travel time. GA reached $82.95 \%$ coverage in 50.60 min, TS did $83.20 \%$ in $3.73 \mathrm{~min}$, and SA did $80.17 \%$ in $52.42 \mathrm{~min}$, while the existing coverage only reaches $73.82 \%$.
\end{abstract}

